\title{
Thyrotoxicosis after COVID-19 vaccination
}

\author{
Rujittika Mungmunpuntipantip ${ }^{1}$ - Viroj Wiwanitkit ${ }^{2}$
}

Received: 14 October 2021 / Accepted: 19 October 2021 / Published online: 29 October 2021

(C) The Author(s), under exclusive licence to Springer Science+Business Media, LLC, part of Springer Nature 2021

\section{Dear Editor,}

We would like to share ideas on the publication "Thyrotoxicosis after COVID-19 vaccination: seven case reports and a literature review" [1]. Lee et al. concluded that "COVID-19 vaccines can cause not only destructive thyroiditis but also AIT... relationship between COVID-19, vaccines, and thyroid diseases" [1]. We agree that the postCOVID-19 vaccination thyroid problem might occur and there might be an association with pathological/abnormal immune response to vaccine. In the present study, a change of thyroxine level is well demonstrated and there is a trend of decreasing when time passes. After vaccination, there is also another important process that might contribute to thyrotoxicosis occurrence. The vaccine can induce increased blood viscosity [2]. The association between hyperviscosity and thyrotoxicosis is proposed [3]. An increasing of blood viscosity is due to rapid increased level of antibody after vaccination and the viscosity will decrease when there is a decline of antibody level. This pattern is the same as observed alteration of thyroid hormone level in the present report by Lee et al. [1]. Finally, if thyroid hormone test is based on immunoassay, an interference, a false aberration of hormone level might be induced by a high blood viscosity [4].

\section{Compliance with ethical standards}

Conflict of interest The authors declare no competing interests.

Publisher's note Springer Nature remains neutral with regard to jurisdictional claims in published maps and institutional affiliations.

\section{References}

1. K.A. Lee, Y.J. Kim, H.Y. Jin, Thyrotoxicosis after COVID-19 vaccination: seven case reports and a literature review. Endocrine. 1-3 (2021). https://doi.org/10.1007/s12020-021-02898-5

2. B. Joob, V. Wiwanitkit, Viscosity after COVID-19 vaccination, hyperviscosity and previous COVID-19. Clin. Appl. Thromb. Hemost. 27, 10760296211020833 (2021)

3. Y.L. Bussmann, M.L. Tillman, A.S. Pagliara, Neonatal thyrotoxicosis associated with the hyperviscosity syndrome. J. Pediatr. 90 (2), 266-268 (1977)

4. C. Selby, Interference in immunoassay. Ann. Clin. Biochem. 36(Pt 6), 704-721 (1999)

Rujittika Mungmunpuntipantip

rujittika@gmail.com

1 Private Academic Consultant, Bangkok, Thailand

2 Dr DY Patil University, Pune, India 